\title{
Proximal intentional neglect: a case study
}

\author{
Michael Gold, Jeffrey Shuren, Kenneth M Heilman
}

\begin{abstract}
Although neglect has been demonstrated in the horizontal, vertical, and radial planes of space and has been attributed to sensory-attentional, motor-intentional, and representational deficits, motor intentional neglect in the radial plane has not been previously described. A patient who had a right parietal infarct was tested with a modified cancellation task that uses a fixed window, thereby controlling attentional demands and allowing one to dissociate between intentional and representational neglect. This patient showed proximal (radial) intentional neglect (a failure to move towards or in proximal space). Unlike controls whose search times decreased with increasing window size, our patient showed no change in search time as a function of window size. This pattern of behaviour suggests that the patient's search strategy does not incorporate factors such as the relation between a fixed target space and a variably sized aperture, but rather was based on the allocation of a certain amount of resources for a fixed period of time after which, regardless of performance, the patient would stop searching.
\end{abstract}

(F Neurol Neurosurg Psychiatry 1994;57:1395-1400)

Neglect is diagnosed clinically on the basis of failure to report, respond, or orient to novel or meaningful stimuli. ${ }^{1}$ From tasks such as line bisection and target cancellation, neglect has been demonstrated in the horizontal (left/right), ${ }^{23}$ vertical (up/down), ${ }^{45}$ and radial (near/far) ${ }^{56}$ planes of space.

Spatial neglect has been attributed to atten-

Department of

Neurology, University of Florida College of

Medicine and the

Neurology Service, Department of Veterans Affairs Medical Center, Gainesville, Florida 32608-1197, USA

M Gold

J Shuren

K M Heilman

Correspondence to: Dr K M Heilman, P O Box 100236, JHMHC, University of Florida, Gainesville,

FL 32610-0236, USA.

Received 22 November 1993 and in revised form 20 April 1994.

Accepted 25 May 1994 tional (sensory), intentional (motor), or representational deficits. According to the attentional hypothesis, patients with spatial neglect may be unaware of stimuli, ${ }^{7}$ have biased attention, ${ }^{8}$ or be unable to disengage their attention..$^{910}$ Because some patients with spatial neglect also fail to image objects in certain regions of space, Bisiach and colleagues ${ }^{11} 12$ hypothesised that these patients have a spatial representational defect. The intentional (motor) hypothesis of neglect proposes that patients with spatial neglect may fail to act in or towards a portion of space. ${ }^{1}$

Horizontal hemispatial intentional neglect has been described in animal models of neglect as the result of parietotemporal, ${ }^{13-15}$ frontal, ${ }^{1316}$ thalamic, ${ }^{17}$ and mesencephalic reticular lesions. ${ }^{13}$ Horizontal (primarily) left intentional neglect has been noted in patients with right sided cerebral lesions, either parietal, $^{18}{ }^{19}$ frontal, ${ }^{19-22}$ or basal-ganglionic ${ }^{18}$ in location. The dissociation of attentional mechanisms from intentional mechanisms has been explored by techniques that invert the direction of movement from the hemispace being explored such that exploration of a neglected hemispace depends on movements in the opposite direction. ${ }^{18} 1923-25$ These studies have confirmed that left horizontal motor intentional neglect is an important mechanism of hemispatial neglect.

Proximal radial neglect has been reported in patients with parietal lesions, ${ }^{6}$ and distal radial neglect has been reported in a patient with temporal lesions. ${ }^{5}$ There is at least one case report of proximal (personal) neglect on the basis of a defective representation ${ }^{26}$ and another case report of a combined horizontal and radial neglect, which was also presumed to be representational in nature, ${ }^{27}$ but motor intentional neglect in the radial plane has not been described previously.

Mijovic $^{28}$ developed a technique that could potentially allow one to dissociate attentional, representational, and intentional neglect. With equipment similar to and adopting some of the terminology of that study, we tested the ability of a patient with a right parietal infarct to simultaneously explore right/left hemispace and near/far hemispace. The technique involves the use of a fixed aperture through which a patient can see a single target at a time. The fixed aperture restricts the area of space that the patient needs to attend to during a search and prevents the patient from using an attentional strategy to find targets. Inattention can be measured by recording the number of targets appearing in the aperture that the patient fails to detect. If the aperture successfully restricts attentional demands, one would expect a very low rate of missed targets under the testing condition.

In the direct search task, the patient moves a piece of cardboard with a fixed aperture over a set of stationary targets. Under this condition, proximal movements lead to exploration of the proximal target space, and distal movements lead to the exploration of the distal target space. Likewise, movements to the right lead to the exploration of the right hemispace, and movements to the left lead to exploration of the left hemispace. Before a target appears in the window, the subject has to have performed a motor exploration. If a subject fails to explore an area of space (right/left; near/far), that failure cannot be attributed to 
an attentional defect, but would suggest a motor intentional or representational defect. In the indirect task, the aperture is stationary and the patient moves the targets under the stationary aperture. In this task, the direction of movement is opposite to the region of space being searched. Therefore, proximal movements lead to exploration of distal target space, whereas distal movements lead to exploration of proximal target space. Horizontal movements are also reversed such that movements to the right lead to exploration of left target hemispace and vice versa.

If the patient has a representational defect, the region of space that is no longer represented should be unexplored in both the direct and indirect conditions. In the direct condition this would entail a failure to move towards or in the neglected space, and in the indirect condition, would entail a failure to move away from the neglected space. If the defect is intentional, however, (a failure to move in or towards a given target hemispace), then the subject would fail to move in a given direction regardless of the condition of the search task. In this case, decreased movement in a given direction would lead to decreased exploration of one side of the target space in the direct task and to decreased exploration of the opposite target space in the indirect task. For example, if the patient had proximal radial neglect due to an intentional defect, one would predict that the patient would miss proximal targets in the direct task and distal targets on the indirect task. If the patient had proximal radial neglect due to a representational defect, however, then one would predict that proximal targets would be missed under both testing conditions.

As there are reports of patients with neglect whose performance on tasks may vary with the vertical or radial position of the stimuli, ${ }^{29} 30$ tasks designed to investigate hemispatial neglect should ideally allow the patient to move freely and should permit the analysis of performance in multiple spatial dimensions simultaneously. The aperture technique described satisfies both criteria. Because the patient's movements are not restricted, the pattern of searching presents a more realistic model of actual behaviour than a task that limits the patient to a single direction of movement (for example, line bisection) and may inadvertently redirect attention or motor intention. Secondly, as the target space is two dimensional, performance can be evaluated in either single dimensional or two dimensional space simultaneously and can reveal defects in more restricted areas of space.

\section{Case report}

A 74 year old, right handed man was admitted to the University of Florida Shands Teaching Hospital with a complaint of left facial weakness and dysarthria. The patient had the onset of progressive left leg weakness one week before and weakness of his left arm two days before admission. At the time of admission, he also became aware of disorientation while driving in a familiar area. There was no history of headache or visual phenomena preceding the weakness. At the time of admission he was noted by his family to be unusually somnolent. There was no history of focal neurological deficits or of relevant head trauma.

His medical history showed type II diabetes and asymptomatic coronary artery disease. $\mathrm{He}$ underwent a coronary bypass eight years before admission.

Neurological examination at the time of admission showed the patient to be alert and oriented in all spheres, to have normal spontaneous language, and to be able to recall three of three objects after five minutes. No testing for neglect was done on admission. Cranial nerves were normal except for a right central 7 th nerve palsy. Motor examination showed normal tone and bulk. Manual motor testing revealed 5/5 strength throughout except in the left arm and hand where it was in the $3+/ 5$ range. The left arm had a prominent upward drift. Sensory examination showed decreased sensation to fine touch and vibration in the left foot. His reflexes were normal and a left Babinski reflex was elicited.

One day after admission, the patient was noted to deny his weakness, complaining only of some numbness in his left arm. At this time trimodal extinction was noted. Forty eight hours after admission, the patient became acutely confused and combative, requiring restraints and sedation. No metabolic, infectious, or neurological aetiology could be established, and the delirium cleared spontaneously within 72 hours. An initial screening for spatial neglect using a free field target cancellation and horizontal line bisections showed difficulty with left sided and proximal targets.

Computed tomography performed 48 hours after admission confirmed the presence of a wedge shaped infarct in the watershed distribution between the right middle cerebral artery and right posterior cerebral artery distributions, involving the posterior portion of the superior temporal gyrus, and extending superiorly to the posterior portion of the supramarginal gyrus and the anterior part of the angular gyrus. Diffuse atrophy and leukoaraosis were noted as well. Medical evaluations failed to produce an aetiology for the stroke. The patient was discharged to a rehabilitation hospital near his home for further physical therapy.

He was admitted to the University of Florida Shands Teaching Hospital's Clinical Research Center four months later to allow for extensive testing. Neurological examination at this time was unchanged.

\section{Procedure}

Preliminary screening for radial neglect was carried out with line bisection ${ }^{1}$ and letter cancellation tasks $^{30}$ to verify the existence of neglect.

The letter cancellation task was carried out in the horizontal (six trials) and vertical (five trials) planes of space. Each target sheet 
contained 56 randomly distributed letters Performance was measured as the percentage of targets missed in each of the two complementary (right/left, near/far) hemispaces.

Line bisection tasks were carried out along the radial axis under two conditions (direct and indirect) with three different line lengths $(203 \cdot 2,254$, and $304.8 \mathrm{~mm})$. In the direct condition (free vision), the patient was able to see the line as well as his hand during the task. In the indirect condition the patient was asked to bisect an unseen line placed on the underside of a board that prevented him from seeing his own hand. To provide the patient with a visual reference in relation to the target line, a second line identical to the target was placed on the top of the board. Bisections were measured to the nearest $\mathrm{mm}$ and scored as a percentage of deviation from the mid-point such that a positive error indicated a distal bias.

For the direct and indirect motor search tasks, 15 black circular targets $5 \mathrm{~mm}$ in diameter were printed in a pseudorandom fashion on an $8.5 \times 14$ inch sheet of paper. A single target in the centre was used as the starting point for a motor search task. None of the targets were within 2 inches of each other and only one target could be seen within the window at a given time. Four equivalent versions of this target sheet were produced by rotation along the $x$ axis and the $y$ axis. The time to complete each search was measured from the time the patient moved away from the central target until he indicated that he was done. No time limits were imposed on the tasks.

In the direct motor search task the target sheet was covered with one of three $24 \times 36$ inch white pieces of cardboard. Each cardboard had a single $1 \times 1,1.44 \times 1.44$, or $2 \times 2$ inch square window in the centre and a handle that had been attached at the midline of the cardboard. The patient was told that he would see a cardboard with a window cut out from it and a black dot in the window, that he was to use the handle to move the window around, and that he was to find as many dots as he could. He was also instructed to say when he saw a target so that it could be marked. Finally, he was instructed to indicate when he thought there were no more targets.

Randomly selected sheets of targets were placed on a table in front of the patient such that the centre target coincided with the patient's mid-sagittal plane. The patient was told to keep his eyes closed until instructed otherwise. A randomly selected aperture was placed over the target sheet so that only the centre target was visible. The handle of the cardboard was then placed in the patient's right hand and he was instructed to open his eyes. The patient then moved the cardboard over the surface of the table indicating when he had seen a target and when he was finished. During the initial testing session, eight trials with each of the window sizes were carried out for a total of 24 trials. During a second session five more trials were carried out to determine if the original pattern of motor searching was still present. The total number of trials was 10 for windows 1 and 3 and nine for window 2 .

The indirect search task used a $24 \times 36$ inch piece of plywood from which either a $1 \times 1$ or a $2 \times 2$ inch square window had been cut out. The plywood cover stood $2-3$ inches above the surface of the table. An $8.5 \times 14$ inch platform set on rollers and to which a handle was attached could be passed under the window, allowing the patient to explore the entire $8.5 \times 14$ inch target space. The same sets of targets used in the direct task were placed on the platform, and the patient was given essentially the same set of instructions. He was told to use the handle to bring the black dots under the window. He was told to search until he thought there were no more targets left. This task was administered a total of 12 times in each of the two window conditions for a total of 24 trials.

To test the effect of the differently sized apertures on motor search times, the direct task was administered to four age and sex matched controls. Each control was tested a total of 12 times.

\section{Statistical methods}

For the letter cancellations, the percentages of targets found in the right and left hemispaces were compared with each other by Wilcoxon paired rank sum test with correction for ties as well as being compared with previously published normal controls. ${ }^{31}$ For the radial line bisection tasks, the patient's performance in the direct condition was compared with his performance in the indirect condition.

For the motor search tasks, the number of targets missed in each quadrant was counted. The total number of targets in the distal, proximal, right, and left sides of the target sheets were the dependent variables and were analysed by Wilcoxon paired rank sum test. Kruskall-Wallis tests with correction for ties were used to compare the total number of errors, the total search time, and the difference in horizontal and vertical errors across the different window sizes on the direct motor search task. Mann-Whitney U tests with correction for ties were used to compare the total number of errors, total search time, and the difference in horizontal and radial errors on the indirect motor search task. To test the effect of the size of the aperture on search times in normal controls, a one way repeated measures analysis of variance (ANOVA) was performed with aperture size as the within subject factor.

\section{Results}

The letter A cancellation tasks were carried out in both horizontal and vertical planes. The patient missed 23.8 (SD 34.2 ) $\%$ of the right sided $v 39.3(25 \cdot 0) \%$ of the left sided targets and missed $26.1(26.9) \%$ of distal $v$ $37 \cdot 8(32.4) \%$ of proximal targets in the horizontal plane. In the vertical plane, the patient missed $32 \cdot 1$ (SD 20.4 ) \% of right sided $v 46.6$ $(21 \cdot 2) \%$ of left sided targets. The patient 
missed more targets on the left side than on the right side ( $\mathrm{n}=11, t=8.5, \mathrm{p}<0.05)$ and missed more targets proximally than distally ( $\mathrm{n}=6, t=0, \mathrm{p}<0.05$ ). The patient clearly performed well below normal controls who averaged $97 \%$ accuracy. ${ }^{31}$

In the radial line bisection task the patient had a mean error of $4.79(4.52) \%$ in the direct condition and had a mean error of 7.03 $(9 \cdot 11) \%$ in the indirect condition. The two conditions were not statistically different (table 1).

In the direct motor search task the patient missed more targets proximally $(44.3 \%)$ than distally $(26 \cdot 1 \%)(t=16$, critical value $=75$, $\mathrm{p}<0.005, \mathrm{n}=26$, one tailed). The patient missed more targets on the left side $(36.5 \%)$ than on the right side (34\%); however, this was not statistically significant $(t=109 \cdot 5, \mathrm{n}=$ 22 , critical value $=75, \mathrm{p}>0 \cdot 10)$. There was no effect of window size on either the total number of targets missed $(H=1 \cdot 218, \mathrm{n}=29$, $\mathrm{df}=2, \mathrm{p}>0.05)$ or the time for searching $(H=2 \cdot 3493, \mathrm{n}=29, \mathrm{df}=2, \mathrm{p}>0 \cdot 05)$. Also, there was no effect of window size on the number of horizontal errors $(H=0.6963$, $\mathrm{df}=2, \mathrm{p}>0.05)$ or on radial errors $(H=$ $0.7346, \mathrm{df}=2, \mathrm{p}>0.05)$. Of a total of 240 targets that appeared in the window, only two were not reported to the examiner $(0 \cdot 83 \%)$.

In the indirect motor search task the patient missed more targets distally $(46.4 \%)$ than proximally $(26 \cdot 8 \%)(t=28, \mathrm{n}=21$, critical value $=42, \mathrm{p}<0.005)$, and he missed more targets on the left side $(51 \cdot 2 \%)$ than on the right side $(19.6 \%)$ of the target space $(t=$ $12 \cdot 5, \mathrm{n}=22$, critical value $=48, \mathrm{p}<0.005$ ) Window size did not have an effect on the time for searching $(U=46$, critical value $=33$ $\mathrm{p}>0.05)$, horizontal errors $(U=47$, critical value $\left.\left(n_{1}=12, n_{2}=12\right)=37, p>0.05\right)$, or radial errors $\left(U=51 \cdot 5\right.$, critical value $\left(n_{1}=12\right.$, $\left.n_{2}=12\right)=37, p>0.05$ ); however it did have an effect for the total number of targets missed $(U=9$, critical value $=27, \mathrm{p}<0.01)$.

The ANOVA of the search times in normal controls showed a significant main effect of window size $(F(2,6)=38.74, \mathrm{p}<0.001$; table $2)$. The repeated measures analysis also

Table 1 Line bisection data

\begin{tabular}{llll}
\hline Condition & Length $(\mathrm{mm})$ & Error (\%) & $S D(\%)$ \\
\hline (n=10): & & & \\
Direct & $203 \cdot 2$ & $6 \cdot 02$ & $4 \cdot 77$ \\
Direct & $254 \cdot 0$ & $5 \cdot 02$ & $4 \cdot 68$ \\
Direct & $304 \cdot 8$ & 3.31 & $4 \cdot 14$ \\
Indirect & $203 \cdot 2$ & $5 \cdot 24$ & $9 \cdot 37$ \\
Indirect & $254 \cdot 0$ & $11 \cdot 06$ & $9 \cdot 18$ \\
Indirect & $304 \cdot 8$ & $4 \cdot 79$ & $8 \cdot 28$ \\
Overall (n=30): & & & \\
Direct & & $4 \cdot 79$ & $4 \cdot 52$ \\
Indirect & & $7 \cdot 03$ & $9 \cdot 12$ \\
\hline
\end{tabular}

Table 2 Times for direct target searches

\begin{tabular}{llcl}
\hline \multirow{4}{*}{ Window size } & \multicolumn{4}{l}{ Mean search time in seconds (SD) } \\
\cline { 2 - 4 } & $1 \times 1$ inch & $1.44 \times 1.44$ inch & $2 \times 2$ inch \\
\hline Controls & $77.75(18 \cdot 2)$ & $60.50(11 \cdot 70)$ & $49.44(8.92)$ \\
Subject & $104.8(47 \cdot 3)$ & $101.44(38.7)$ & $89.3(21.5)$ \\
\hline
\end{tabular}

showed that the difference in search times between each of the windows was statistically significant $(1 \times 1 v 1.44 \times 1.44$ inch $(F(2,6)=$ $18.68, \mathrm{p}<0.05) ; 1 \times 1 v 2 \times 2$ inch $(F(2,5)=$ $71 \cdot 16, \mathrm{p}<0.05), 1.44 \times 1.44 \quad v 2 \times 2$ inch $(F(2,5)=28.22, \mathrm{p}<0.05))$. Analysis of the control data showed that less time was required to search the target space as the size of the window increases. By contrast, the ANOVA performed on the patient showed no effect of window size on search times $(F(2,17)$ $=1 \cdot 02, \mathrm{p}=0.3823$ ).

\section{Discussion}

The results of clinical examinations as well as of the screening tests for neglect suggested that this patient had a mild neglect of left hemispace and near or proximal peripersonal space.

Errors in traditional cancellation tasks may be related to attentional, intentional, or representational deficits. Because targets that appeared within the window were rarely unattended, the results of the motor search tasks cannot be accounted for by defects in spatial attention, but rather suggest intentional or representational deficits.

The results of the motor search tasks indicate that this patient missed proximal targets in the direct condition and distal targets in the indirect condition. This pattern of errors agrees with our predicted results in the case of motor intentional neglect and indicates that the patient had difficulty exploring peripersonal space and failed to move his arm towards his body (proximal directional hypokinesia or hypometria).

In the horizontal (right/left) dimension our results are less clear. If the patient had a representational deficit of left horizontal hemispace, we would expect him to miss left sided targets in both direct and indirect motor search tasks. If the patient had an intentional deficit he should miss left sided targets on the direct task and right sided targets on the indirect task. The patient missed more targets on the left side on the direct task and failed to explore the left side on the indirect task, suggesting a mild representational defect. The lack of a statistically significant result on the direct task, however, precludes a definitive answer.

Whereas our results show that the patient, who had a right parietal infarct, has proximal intentional neglect and a mild left hemispatial representational neglect, Mijovic $^{28}$ was unable to find intentional neglect. Although both studies used the aperture technique, there are important differences between them. Mijovic measured the time required to find a single target, and in several conditions, provided the subjects with targets that incorporated spatial or directional cues, and did not analyse the data for radial neglect.

The results of our study require us to consider two issues. Firstly, in most previous reports of intentional neglect, patients had frontal lesions. Our patient had intentional neglect associated with a parietal lesion, 
however. Secondly, our patient had a dissociation between two different forms of neglect, representational and intentional neglect in the horizontal and radial planes (a representational defect in the horizontal plane but not the radial plane and had an intentional defect in the radial plane but not in the horizontal plane).

Hemispatial neglect is classically attributed to damage to the posterior part of the right hemisphere $^{3}$ and is thought to be due to damage to a distributed cortico-limbicthalamic-reticular network. ${ }^{1223233}$ This network includes cortical (parietal, frontal, and cingulate) and subcortical regions (thalamus, basal ganglia, ${ }^{18}$ and mesencephalon). ${ }^{131}$ Although earlier studies suggested that the parietal lobe had a specialised function in spatial mapping, ${ }^{34-38}$ and that the frontal lobes had a special role in motor planning, ${ }^{39} 40$ more recent studies support a network in which parietal and frontal lobes function both in spatial mapping ${ }^{1641-45}$ and in motor planning. ${ }^{16} 4146-49$ Single cell recordings and ablation studies of both parietal and frontal lobes in animals have also identified specific cell populations and regions responsive to stimuli or related to movement in specific spatial planes. ${ }^{3441} 42454649-51$ Also, studies of the connectivity between the parietal and frontal lobes have shown multiple direct parallel projections, ${ }^{4952}$ some reciprocal in nature ${ }^{53}$ and some that may be mediated by other cortical or subcortical structures. ${ }^{415455}$ The pattern of termination of the parietal axons within the prefrontal cortex indicates a feed forward mechanism, whereas the pattern of termination of prefrontal axons within the parietal cortex is consistent with a feedback mechanism. ${ }^{53}$

The demonstration that the parietal lobe has functions associated with motor planning and the identification of regions with spatial specificity can help explain the intentional nature and the spatial selectivity of the patient's deficit. To account for the sparing of proximal radial representation maps, one would have to postulate that these maps are either bihemispheric or that there is an anatomical separation of the representations of horizontal from radial space in the right parietal lobe analogous to the segregation identified for visual attention in horizontal hemispace. ${ }^{56}$

Although the patient's representations of peripersonal space were intact, the neural net embodying these representations was unable to activate the areas that mediate planning for specific motor acts in a given region of space. By contrast with the radial representations, our patient's lesion seemed to damage the left horizontal representational maps, suggesting that these may be more unilaterally represented. Because our data suggest that radial representational maps are separate from horizontal representational maps, the mechanisms underlying neglect on the basis of representational defects in the horizontal plane may be different from those in the radial plane.

We examined the amount of time the patient devoted to a given search task and compared it with age and sex matched controls. We postulated that the amount of time expended in exploration is proportional to both the space to be searched and the window used to explore that field. Although we confirmed that search times decreased with larger window sizes in normal controls, no such relation was identified in our subject. In fact, he devoted equivalent amounts of time to tasks with windows that progressively doubled in area. Window size may have an influence on the efficiency of motor searches; however, because in this paradigm only one target could be seen even with the largest window, it is possible that window size would not affect search times or accuracy. The fact that normal controls showed a robust window effect suggests that the lack of a window effect on the part of the subject was not an artifact of the paradigm. Additionally, both extremes of behaviour were seen, with the patient having very short searches with a small window and prolonged searches with a large window. These data suggest that the patient's strategy for searching did not incorporate factors such as the relation between a fixed target space and a variably sized aperture, but rather was based on the allocation of a certain amount of resources for a fixed period of time after which, regardless of performance, the patient would stop searching.

The results of this study show a proximal radial intentional neglect and a simultaneous left representational hemispatial neglect associated with a right parietal infarct. The coexistence of deficits in two orthogonal spaces on the basis of two separate mechanisms supports the theory that attentional, representational, and intentional mechanisms are mediated by parallel networks segregated by the region of space to which attention is to be directed or in which some action is to be taken.

This work was supported by the Medical Research Service of the Department of Veteran Affairs, grant GCRC, MO1 RR0082 from the National Institute of Health, and the Memory Disorder Clinic, Florida Department of Elder Affairs, Alzheimer's Disease Initiative. We thank Ms Barbara Haws Alzheimer's Disease Initiative. We thank Ms Barbara Haws
(VA Research Service) for her assistance in constructing the testing apparatus.

1 Heilman KM, Watson RT, Valenstein E. Neglect and related disorders. In: KM Heilman, $\mathrm{E}$ Valenstein, eds. Clinical neuropsychology. 2nd ed. New York: Oxford University Press, 1985:243-93.

2 Halligan PW, Marshall PC. How long is a piece of string? A study of line bisection in a case of visual neglect. Cortex 1988;24:321-8.

3 Brain WR. Visual disorientation with special reference to lesions of the right cerebral hemisphere. Brain 1941;64: lesions 71 .

4 Rapcsak SZ, Cimino CR, Heilman KM. Altitudinal neglect. Neurology 1988;38:277-81.

5 Shelton PA, Bowers D, Heilman KM. Peripersonal and vertical neglect. Brain 1990;113:191-205.

6 Mennemeier M, Wertman E, Heilman KM. Neglect of near peripersonal space. Brain 1992;115:37-50.

7 Riddoch MJ, Humphreys G. The effect of cuing on unilateral neglect. Neuropsychologia 1983;21:589-99.

8 Kinsbourne M. A model for the mechanism of unilateral neglect of space. Transactions of the American Neurological Association 1970;95:143.

9 Posner MI, Walker JA, Friedrich FJ, Rafal RD. Effects of parietal injury on covert orienting of attention. f Neuroscience 1984;4:1863-74.

10 Mark VW, Kooistra CA, Heilman KM. Hemispatial neglect affected by non-neglected stimuli. Neurology 1988;38:1207-11.

11 Bisiach E, Luzzatti C. Unilateral neglect of representational space. Cortex 1978;14:129-33. 
12 Bisiach E, Luzzatti C, Perani D. Unilateral neglect, representational schema and consciousness. Brain 1979;102 609-18.

13 Watson RT, Miller BD, Heilman KM. Nonsensory neglect. Ann Neurol 1978;3:505-8.

14 Valenstein E, Heilman KM, Watson RT, Van Den Abell $\mathrm{T}$. Nonsensory neglect from parietotemporal lesions in monkeys. Neurology 1982;32:1198-201.

15 Watson RT, Valenstein E, Day A, Heilman KM. Normal tactile threshold in monkeys with neglect. Neurology 1986;36:636-40.

16 Rizzolatti G, Matelli M, Pavesi G. Deficits in attention and movement following the removal of postarcuate (area 6) and prearcuate (area 8) cortex in macaque monkeys. and prearcuate (area 8)

17 Valenstein E, Heilman KM. Unilateral hypokinesia and motor extinction. Neurology 1981;31:445-8.

18 Heilman KM, Bowers D, Coslett HB, Whelan H, Watson RT. Directional hypokinesia: prolonged reaction times for leftward-movement in patients with right hemispher lesions and neglect. Neurology 1985;35:855-9.

19 Coslett HB, Bowers D, Fitzpatrick E, Haws B, Heilman KM. Directional hypokinesia and hemispatial inattention in neglect. Brain 1990;113:475-86.

20 Heilman KM, Valenstein E. Frontal lobe neglect in man Neurology 1972;22:660-4.

21 Daffner KR, Ahern GL, Weintraub S, Mesulam MM Dissociated neglect behaviour following sequential strokes in the right hemisphere. Ann Neurol 1990;28: 97-101.

22 Mesulam MM. A cortical network for directed attention and unilateral neglect. Ann Neurol 1981;10:309-25.

23 Meador KJ, Watson RT, Bowers D, Heilman KM Hypometria with hemispatial and limb motor neglect. Brain 1986:109:293-305.

24 Tegner $R$, Levander $M$. Through a looking glass: A new technique to demonstrate directional hypokinesia in unilateral neglect. Brain 1991;114:1943-51.

25 Bisiach E, Gemiani G, Berti A, Russoni ML. Perceptual and premotor factors of unilateral neglect. Neurology 1990;40:1278-81.

26 Guariglia C, Antonucci G. Personal and extrapersonal space: A case for neglect dissociation. Neuropsychologia 1992;30:1101-9.

27 Halligan PW, Marshall JC. Left neglect for near, but not far space in man. Nature 1991;350:498-500.

28 Mijovic D. Mechanisms of visual spatial neglect. Absence of directional hypokinesia in spatial exploration. Brain 1991;114:1575-93.

29 Halligan PW, Marshall JC. Is neglect (only) lateral? A quadrant analysis of line cancellation. $\mathcal{f}$ Clin Exp quadrant

30 Weintraub S, Mesulam MM. Visual hemispatial inattention: stimulus parameters and exploratory strategies. f Neurol Neurosurg Psychiatry 1988;51:1481-8.

31 Ferro JM, Kertesz A, Black SE. Subcortical neglect: quantitation, anatomy and recovery. Neurology 1987;37: $1487-92$

32 Heilman KM, Valenstein E. Mechanisms underlying hemispatial neglect. Ann Neurol 1979;5:166-70.

33 Watson RT, Valenstein E, Heilman KM. Thalamic neglect: possible role of the medial thalamus and nucleus reticularis in behavior. Arch Neurol 1981;38:501-6.

34 Bruce CJ, Desimone R, Gross CG. Visual properties of neurons in a polysensory area in superio temporal sulcus of the macaque. $f$ Neurophysiol 1981; temporal sulc

35 Colby CL, Gattass R, Olson CR, Gross CG. Topographic organization of cortical afferents to extrastriate area PO in macaque: a dual tracer study. 7 Comp Neurol 1988 . 296:392-413.
36 Ungerleider LG, Desimone R. Cortical connections of visual area MT in the macaque. $f$ Comp Neurol 1986; 248:190-222.

37 Seagraves MA, Goldberg ME. Functional properties of corticotectal neurons in the monkey's frontal eye field. f Neurophysiol 1987;58:1387-419.

38 Grafton ST, Mazziotta JC, Woods RP, Phelps ME. Human functional anatomy of visually guided finger movements. Brain 1992;115:565-87.

39 Freund HJ. Premotor areas in man. Trends Neurosci 1984; 7:481-3.

40 Orgogozo JM, Larsen B. Activation of the supplementary motor area during voluntary movements in man suggests motor area during voluntary movements in man suggests

41 Goldman-Rakic P. Topography of cognition: parallel distributed networks in primate association cortex. Ann Rev tributed networks in primate

42 Schwartz AB, Kettner RE, Georgopoulos AP. Primate motor cortex and free arm movements to visual targets in three dimensional space. I. Relations between single cell discharges and direction of movement. $\mathcal{F}$ Neurosci 1988;8:2913-27.

43 Seagraves MA, Goldberg ME. Functional properties of corticotectal neurons in the monkey's frontal eye field. f Neurophysiol 1987;58:1387-419.

44 Barone $\mathrm{P}$, Joseph JPO. Prefrontal cortex and spatial sequencing in macaque monkey. Exp Brain Res 1989; 78:447-64.

45 Gentilucci M, Fogassi L, Luppino G, et al. Functional organization of inferior area 6 in the macaque monkey I. Somatotopy and the control of proximal movements. Exp Brain Res 1988;71:475-90.

46 Mountcastle VB, Lynch JC, Georgopoulos A, Sakata H, Acuna C. Posterior parietal association cortex of the
monkey: command functions for operations within monkey: command functions for operations within

47 Deuel RK, Farrar CA. Stimulus cancellation by macaques with unilateral frontal or parietal lesions. Neuropsychologia 1993;31:29-38.

48 Lynch JC. The functional organization of posterior parietal association cortex. Behav Brain Sci 1980;3:485-534.

49 Leinonen L, Hyvarinen J, Nyman G, Linnakoski GI Functional properties of neurons in lateral part of associative area in awake monkeys. Exp Brain Res 1979, 34:299-320.

50 Rizzolatti G, Gentilucci M, Matelli $M$. Selective spatial attention: one center, one circuit or many circuits? In: Posner MI, Marin OSM, eds. Attention and performance XI. Hillsdale NJ: LEA, 1985:251-69.

51 Steinmetz MA, Botter BC, Duffy CJ, Mountcastle VB Functional properties of parietal visual neurons: radial Functional properties of parietal visual neurons: radial
organization of directionalities within the visual field. organization of direction

52 Petrides M, Pandya DN. Projections to the frontal cortex from the posterior parietal region in the Rhesus monkey f Comp Neurol 1984;228:105-16.

53 Schwartz ML, Goldman-Rakic PS. Callosal and intrahemispheric connectivity of the pre-frontal association cortex in rhesus monkey: relation between intraparietal and principal sulcal cortex. F Comp Neurol 1984;226: 403-20.

54 Selemon LD, Goldman-Rakic PS. Longitudinal topography and interdigitation of corticostriatal projections in the rhesus monkey. $\mathcal{F}$ Neurosci 1985;5:776-94.

55 Morecraft RJ, Geula C, Mesulam MM. Architecture of connectivity within a cingulo-fronto-parietal neurocognitive network for directed attention. Arch Neurol cognitive networ

56 Corbetta M, Miezin FM, Shulman GL, Petersen SE. A PET study of visuospatial attention. $\mathcal{F}$ Neurosci 1993;13: 1202-26. 